\title{
Simulation on Flow Behaviors of Viscoelastic Polymer Solution in a Channel with Sudden Contraction
}

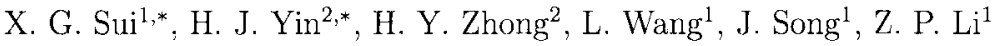 \\ ${ }^{1}$ Oil Recovery Plant No1, Daqing Oilfield Corp. Ltd., Daqing, Heilongjiang 163001, China \\ ${ }^{2}$ Key Laboratory of Enhanced Oil and Gas Recovery of Ministry of Education, Daqing Petroleum \\ Institute, Daqing, Heilongjiang 163318, China
}

\begin{abstract}
In the process of water flooding alone, the residual oil remaining within porous media is difficult to be displaced or recovered. In comparison, polymer flooding is more effective. Experimental results indicated the visco-elasticity of polymer solutions can enhance the displacement efficiency, but there are few theoretical studies on this subject. The viscoelastic fluid can improve the displacement efficiency, which has been proved by experiments.

The Upper-Convected Maxwell (UCM) model has been normally used to describe the rheological properties of polymer solutions. However, this model considers only viscosity and elasticity of a fluid without including the non-Newtonian power law behavior, a very important property to polymer solutions in reservoirs. Therefore, we present a Modified Upper-Convected Maxwell (MUCM) in this paper to take into account this property. In this paper, the steady flow mathematical model of MUCM fluids is established. The MUCM model is applied for describing viscoelastic fluid flow through sudden contraction. The contours of velocity and stream function are drawn and micro-scale sweep efficiency is calculated quantitatively. The various regularities, corresponding to the change in visco-elastic properties of the fluid, are studied. This study will be helpful for the further studying the mechanics of the polymer flooding deeply, at the same time it also should be useful in sclecting polymer fluids and designing polymer flooding operations.

The main conclusions can be concluded:

(1) The MUCM constitutive model more correctly describes rheology of polymer solutions, because the viscosity, elasticity and power law properties of the polymer solutions are all considered.

(2) Numerical results show the visco-elasticity of polymer solutions is the main factor influencing sweep efficiency. With increasing elasticity, the flowing area in the dead end of pores is enlarged significantly, thus the area with immobile zones becomes smaller.

(3) Flow velocity is much larger than that for a Newtonian fluid, the sweep area and displacement efficiency increase as the elasticity increases. The visco-elastic nature of the displacing polymer fluids can in general improve the displacement efficiency in pores compared to using Newtonian fluids. This conclusion should be useful in selecting polymer fluids and designing polymer flooding operations.
\end{abstract}

\section{REFERENCES}

1. Wang D M, Cheng J C, Yang Q Y, et al. Viscous-elastic polymer can increase in cores. SPE 63227. 2000: $2-8$

2. Yin H J, Wang D M, Zhong H Y. Study on flow behaviors of viscoelastic polymer solution in micropore with dead end. SPE 101950, 2006: 1-10

3. Crochet M J, Davies A R, Walters K. Numerical Simulation of non-Newtonian Flow. New York: Elsevier Science Publisher B. V. 1984 